55, 2, pp. 393-406, Warsaw 2017

DOI: $10.15632 /$ jtam-pl.55.2.393

\title{
FAULTS DETECTION IN GAS TURBINE ROTOR USING VIBRATION ANALYSIS UNDER VARYING CONDITIONS
}

\author{
Benrabeh Djaidir, Ahmed Hafaifa, Abdallah Kouzou \\ Applied Automation and Industrial Diagnostics Laboratory, Faculty of Science and Technology, University of Djelfa, Algeria \\ e-mail:b.djaidir@univ.djelfa.dz; hafaifa.ahmed.dz@ieee.org; kouzouabdellah@ieee.org
}

\begin{abstract}
Monitoring of rotating machines is a very important task in most industrial sectors, which requires a chosen number of performance indicators during the exploitation of such kind of equipments. Indeed, for understanding the undesirable phenomena complexity of the industrial systems under operation, a reliable and an accurate mathematical modeling is required to ensure the diagnosis and the control of these phenomena. This work proposes development of a fault monitoring system of a gas turbine type GE MS 3002 based on vibration analysis technique using spectral analysis tools. The obtained results prove the effectiveness of the presented monitoring tool approach which is applied on the gas turbine, for avoiding the operation under vibration mode and for generating optimal performance during the exploitation of the gas turbine.
\end{abstract}

Keywords: default bearings, dynamic behavior, faults detection, gas turbine, monitoring system

\section{Introduction}

Vibration analysis of rotating machines in industrial plants is widely used to ensure premature faults diagnosis before these machines may break down. Gas turbines are the most important pivotal rotating machines belonging to the family of internal combustion engines that are used intensively in oil and gas industrial plants. Indeed, it is used to achieve the main function of generating mechanical energy in form of shaft rotation from kinetic energy of gases produced in the combustion chamber (Djaidir et al., 2015, 2016; Djeddi et al., 2015, 2016; Eshati et al., 2013; Jurado and Carpio, 2006; Kim et al., 2011). However, these machines are subject to some phenomena inducing important vibrations that can be very critical to their mechanical state.

The work presented in this paper proposes a supervision approach based on vibration analysis, to ensure a permanent fault diagnosis of the studied gas turbine, where the main aim is to prevent failures that may cause malfunctions and to fulfill an optimal operation availability of such a machine. Indeed, there are several methods that have been used for characterization and modeling of the gas turbine parameters. However, these methods are less exploitable for the control of such systems due to its complexity. The proposed approach in this work is based on the analysis of the magnitude and the harmonics spectrum of signals resulting from measured vibrations on the gas turbine under different failures that may affect this kind of machine during its normal operation.

Gas turbines are widely used in many industrial applications such as oil industry where they are mainly used for wells re-injection and gas transportation along the pipelines. These machines are subject to various instability factors that are influencing directly their operating performances. Therefore, in order to improve the performances of these systems, many researches previously built mathematical models that represent the real time operation state more accurately and instantaneously. Madhavan et al. (2014) developed a vibration model based on 
damage detection of rotor blades in a gas turbine engine. They showed that the developed model can be used to carry out the analysis of mechanical components failures detection of the system under study. Liu et al. (2015) studied the influence of large scale wind turbine blade vibration based on the influence of the aerodynamic effects. Usman et al. (2015) presented the impact of operating conditions and exhaust gas recirculation on the performance of rotors dynamics of a gas turbine system. However, the current development of new technologies has allowed researchers and industrial practices to improve the performance of gas turbines and to contribute effectively to developing new methods and algorithms for controlling of such systems. In this context, many industrial monitoring systems are being developed by several researchers to ensure fault detection, as well as for the diagnosis of defects (Ali et al., 2015; Djaidir et al., 2015, 2016; Djeddi et al., 2015, 2016; Günyaz, 2013; Krzyzynski et al., 2000; Liu et al., 2015; Sanaye and Tahani, 2010; Simani and Patton, 2008).

The present paper deals with the modeling of gas turbine defects based on vibration analysis using mathematical techniques, where a number of assumptions representing different operating parameters of the studied gas turbines have been taken into account. In this paper, the analysis of dynamic behavior of rotor vibration of the gas turbine type GE MS 3002 is proposed. This gas turbine rotates at high speed up to $7100 \mathrm{tr} / \mathrm{min}$ and it is supported by bearings that may be subject to faults during normal operation, which may contribute directly to the deterioration of the gas turbine in a rapid manner.

The obtained in this paper results show clearly the effectiveness of the presented modeling of the defects in a gas turbine. The presented approach allows one to build a diagnostic strategy based on the vibration behavior analysis. On the other side, the experimental procedure for tracking the anomalies in the gas turbine is presented. It is based on the use of the obtained vibration signal spectrum and the associated analysis.

\section{Dynamic vibration behavior in gas turbine}

Gas turbines are heavily utilized in industry, where they are used mainly for producing and ensuring important mechanical energy (Ford et al., 2013; Guasch, 2013; Lee et al., 2013; Rahmoune et al., 2015). They are used for driving of fixed appliances where important output power is required such as in electrical power generator, in powerful compressors, in powerful pumps, etc. Therefore, the analysis of dynamic behavior of the gas turbine is attracting much attention from the researcher and industrialists. It is based on the study of vibration behavior of the main element of the gas turbine which is the high pressure rotor (HP) (Madhavan et al., 2014; Rahmoune et al., 2015).

The vibration signal is presented by the following expression

$$
x(t)=A \sin (w t+\phi)
$$

where $w$ is the frequency of vibration, $\phi$ is the phase and $A$ the amplitude of vibration in micrometers $[\mu \mathrm{m}]$.

The vibration speed $\nu(t)$ and the vibration acceleration $a(t)$ are obtained by differentialing vibration expression (2.1) as follows

$$
\nu(t)=\frac{d x(t)}{d t}=A w \cos (w t+\phi) \quad a(t)=\frac{d \nu(t)}{d t}=-A w^{2} \sin (w t+\phi)
$$

Practically, all units are based on millimeters and seconds.

Based on equations (2.1) and (2.2), the following expressions can be deduced

$$
|x|=\frac{|\nu|}{w}=\frac{|a|}{w^{2}} \quad|\nu|=|x| w=\frac{|a|}{w} \quad|a|=|\nu| w=|x| w^{2}
$$


where $x$ is the displacement, $\nu$ is the vibration speed, $a$ is the vibration acceleration and $w$ is the frequency of vibration.

Equation (2.3) highlights the importance of the choice of physical variables for data measurement and monitoring of the gas turbine. Compared to the model based on speed measurement, the displacement measurements are used to reduce the high and the medium frequency vibration effects, which can amplify the low frequency vibration components. In this case, radial vibrations in the gas turbine are studied in the simple case of a circular cross-section of the rotor in the transverse position.

The dominant torsional displacement is the rotation of the cross sections given by the angular displacement $\alpha$. The simplified displacement is used as follows

$$
\begin{aligned}
& u_{1}\left(x_{1}, x_{2}, x_{3}, t\right) \approx 0 \quad u_{2}\left(x_{1}, x_{2}, x_{3}, t\right) \approx-x_{3} \alpha\left(x_{1}, t\right) \\
& u_{3}\left(x_{1}, x_{2}, x_{3}, t\right) \approx x_{2} \alpha\left(x_{1}, t\right)
\end{aligned}
$$

For the deformation calculations, the Hamilton functional is used

$$
\varepsilon_{11}=\varepsilon_{22}=\varepsilon_{33}=\varepsilon_{23} \quad \varepsilon_{12}=-\frac{1}{2} x_{3} \frac{\partial \alpha}{\partial x_{1}} \quad \varepsilon_{13}=\frac{1}{2} x_{2} \frac{\partial \alpha}{\partial x_{1}}
$$

The Hamilton functional construction is given by the following equation

$$
\begin{aligned}
& H(\alpha)=\int_{t_{0}}^{t_{1}} \int_{0}^{l}\left[\frac{1}{2} \rho I_{1}\left(\frac{\partial \alpha}{\partial t}\right)^{2}-\frac{1}{2} G I_{1}\left(\frac{\partial \alpha}{\partial x_{2}}\right)^{2}+M_{1} \alpha\right] d x_{1} d t \\
& I_{1}=\int_{S}\left(x_{2}^{2}+x_{3}^{2}\right) d x_{2} d x_{3}
\end{aligned}
$$

The rotor motion is given by

$$
\rho I_{1} \frac{\partial^{2} \alpha}{\partial^{2} t^{2}}-\frac{\partial}{\partial x_{1}}\left(G I_{1} \frac{\partial \alpha}{\partial x_{1}}\right)=M_{1} \quad \forall x_{1} \in[0, l], \quad \forall t \in \Re
$$

where $G=E /[2(1+\nu)]$ is the shear modulus of the rotor in the limitat conditions $x_{1}=0$ and $x_{1}=l$

$$
\begin{array}{ll}
\alpha(0, t)=0 & G I_{1} \frac{\partial \alpha}{\partial x_{1}}(0, t)=0 \\
\alpha(l, t)=0 & G I_{1} \frac{\partial \alpha}{\partial x_{1}}(l, t)=0
\end{array}
$$

The conditions in the initial state are given by $\alpha=0$ and the condition $G I_{1}=\partial \alpha / \partial x_{1}=0$ when no torsion at the free end is provided. The stress on the rotor surface is determined by using the following equation

$$
\left[\begin{array}{c}
\sigma_{11} \\
\sigma_{22} \\
\sigma_{33} \\
\sigma_{23} \\
\sigma_{13} \\
\sigma_{12}
\end{array}\right]=\left[\begin{array}{cccccc}
E & \nu E & \nu E & 0 & 0 & 0 \\
\nu E & E & \nu E & 0 & 0 & 0 \\
\nu E & \nu E & E & 0 & 0 & 0 \\
0 & 0 & \frac{E}{2(1+\nu)} & 0 & 0 & 0 \\
0 & 0 & 0 & \frac{E}{2(1+\nu)} & 0 & 0 \\
0 & 0 & 0 & 0 & 0 & \frac{E}{2(1+\nu)}
\end{array}\right]\left[\begin{array}{c}
0 \\
0 \\
0 \\
-x_{3} \alpha_{x_{1}} \\
-x_{2} \alpha_{x_{1}} \\
0
\end{array}\right]
$$

In practice, gas turbines systems are complex and are generally non-stationary. Consequently, their non-linear behavior makes the modeling step of the vibrations very difficult. Hence, implementation of a reliable predictive tool is required to ensure the control and the diagnosis of 
such systems. For this purpose, vibration analysis techniques are used, tested and validated in this paper.

The vibration analysis used is based on the real vibration data collected on the studied gas turbine at different phases of operation. For the present application, the points of measurements are shown in Fig. 1, where a moving accelerometer is used for these measurements for both levels. Indeed, there are three sensors that are used with accelerometers on different positions (horizontal, vertical and axial) at each level, as shown in Fig. 1.

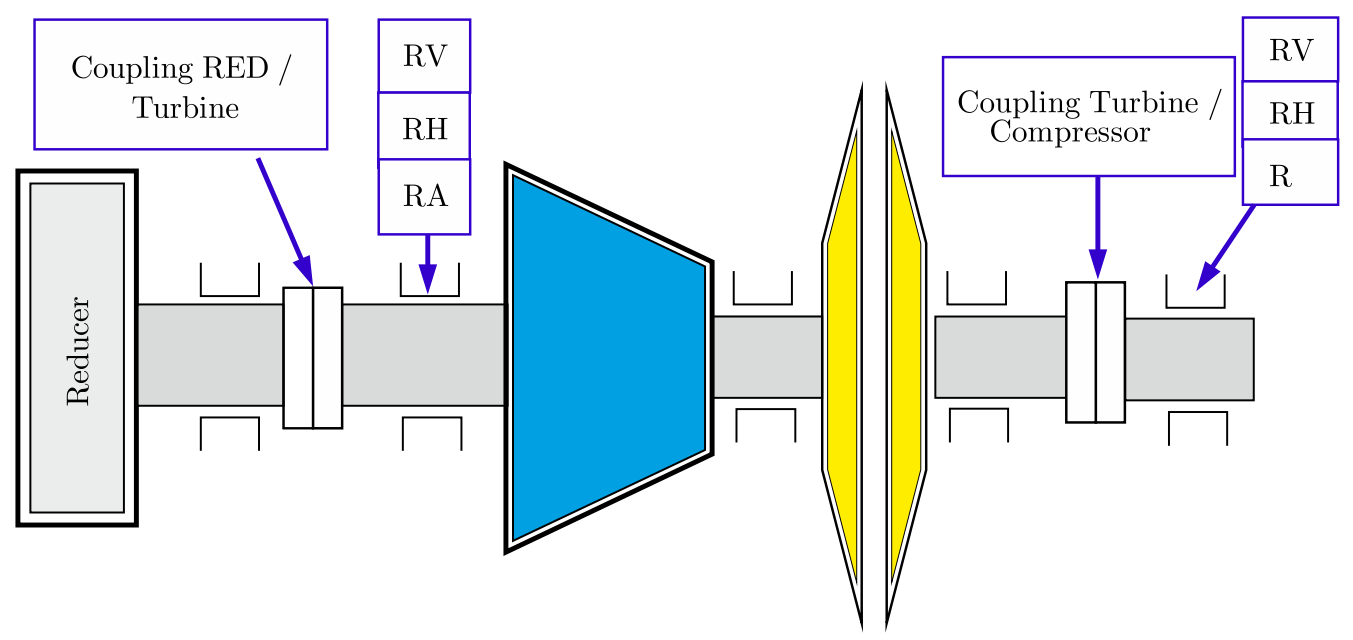

Fig. 1. Experimental installation of the gas turbine system

The recorded vibrations of the studied gas turbine can be quantified by three fundamental quantities. The displacement, the speed and the acceleration. The model considered comprises two entrances, the room temperature and the fuel mass flow rate which is expressed as follows

$$
\dot{m}_{c}=\sqrt{\Delta P} \frac{1}{2} \rho \mu^{2}=\frac{\sqrt{\Delta P}}{k} \Rightarrow \dot{m}_{c}=\frac{1}{k} \sqrt{\Delta P}
$$

The air mass flow $\dot{m}_{a}$ is given by

$$
\begin{aligned}
& \dot{m}_{a}=k^{\prime}\left(P_{2}-P_{1}\right) \\
& \dot{m}_{a}=k^{\prime}\left(P C D-P_{a t m}\right)
\end{aligned}
$$

It is necessary to control the combustion of the gas turbine by regulating the ratio of the efficiency $F$ given by

$$
F=\frac{T_{3}-T_{2}}{\eta_{b} C V+T_{3}}
$$

The sensors are installed on the gas turbine to provide dynamic behavior measurements of the high pressure (HP) turbine shaft speed, the low pressure (BP) turbine shaft speed and the temperature of the blades.

\section{Gas turbine parameters modelling and analysis}

All the rotating machines used in different industries applications are subject to more or less vibrations. These vibrations are due basically to poor distribution of mobile masses, especially in the rotor, which is essentially caused by imperfect machining during their fabrication or a lack of homogeneity of the metal constituting the rotor. Indeed, the origin of vibrations observed in a gas turbine can be caused by different phenomena such as (Galindo et al., 2013; Günyaz, 2013; Krzyzynski et al., 2000): 
- Unbalance of the rotor,

- Rapid braking of the shaft,

- A mechanical failure; such as a broken blade,

- Permanent deformation of the rotor.

The rotating part of the gas turbine consists of two shafts on which several wheels holding the blades are mounted, where the line connection between the bodies of the two shafts is ensured by bearings. Therefore, vibration behavior of such machines extremely depends on these components. It is obvious that each such rotating machine is exposed to dynamic excitations extremely diverse and specific to its mode of operation. At the same time, the fixed and moving parts are vibrating structures, depending on their modal characteristics and their vibration damping capacities. Each part of the system (fixed or mobile) generates dynamic vibration constraints that cause fatigue of the rotating machine parts. It is impossible for a rotating machine which is designed, even with more and more advanced materials, not to vibrate. However, the manufacturers of such machines try to improve the quality of materials and design to built rotating machines that can support more severe vibration constraints.

The research work presented in this paper allows modeling and analysis of dynamic vibration based on the spectral analysis by considering various defects that may occur in this type of machines.

\subsection{Spectral analysis}

The Fourier Transform (FT) can convert a periodical signal into a series sum (which may be infinite) of sine and cosine functions. Suppose a real signal $x(t)$ is defined with a finite energy. The Fourier Transform of the signal $x(t)$ is defined by the following equation

$$
F T_{f}\{x(t)\}=X(f)=\left\langle x(t), \mathrm{e}^{2 \pi \mathrm{i} f t}\right\rangle=\int_{-\infty}^{+\infty} x(t) \mathrm{e}^{-2 \pi \mathrm{i} f t} d t
$$

A discrete signal $x(k)$ of length $N$ presenting the number of values during a fixed interval, can be presented as follows

$$
X(m)=\sum_{k=0}^{N-1} x(k) \mathrm{e}^{-\frac{2 \pi \mathrm{i} m k}{N}}
$$

On the other side, the spectrum of an aperiodic signal is given by its FT

$$
X(\omega)=F T[x(t)]
$$

where $F T[x(t)]$ is spectrum of $x(t)$.

The spectral energy density (SED) in the case of a discrete signal is defined through the discrete Fourier transform as follows

$$
P_{x}(m)=|x(m)|^{2} \quad S_{x}(m)=|X(m)|
$$

It can be shown that the energy of the signal, which is defined as the integral energy transferred at each instant $x(t)$, which can also be expressed as the integral of energy contributions carried by each frequency given by

$$
E=\int_{-\infty}^{+\infty}|x(t)|^{2} d t=\int_{-\infty}^{+\infty}|x(\omega)|^{2} \frac{d \omega}{2 \pi}=\int_{-\infty}^{+\infty}|x(f)|^{2} d f
$$


The energy spectrum of the signal is replaced by the square module of the FT of the square module of $c_{n}$ by the spectrum of energy $|x(\omega)|^{2}=|x(f)|^{2}$ of $x(t)$ with $\lim _{T \rightarrow \infty} T c_{n}=$ $\left|\int_{-\infty}^{+\infty} x(t) \mathrm{e}^{-\mathrm{i} \omega t} d t\right|$

In this paper, the estimation method of the spectral density is proposed for the vibration faults detection and isolation in gas turbine. This method presents a good spectral analysis tool from the point of view of calculation and allows obtaining good results for a large class of signals in the studied gas turbine.

\subsection{Vibration analysis}

As mentioned previously, the gas turbines are subject to unstable phenomena, because the shaft cannot be perfectly balanced. The presence of residual unbalance on the shaft line is counted by adding point masses. The addition of a large unbalance to the shaft is explained by the loss of a blade on one of the disks. The imbalance is characterized by its kinetic energy when the mass $m_{b}$ is located at the point $B$ in the plane of the disk at a distance $d$ from its geometric centre $C$, Fig. 2.

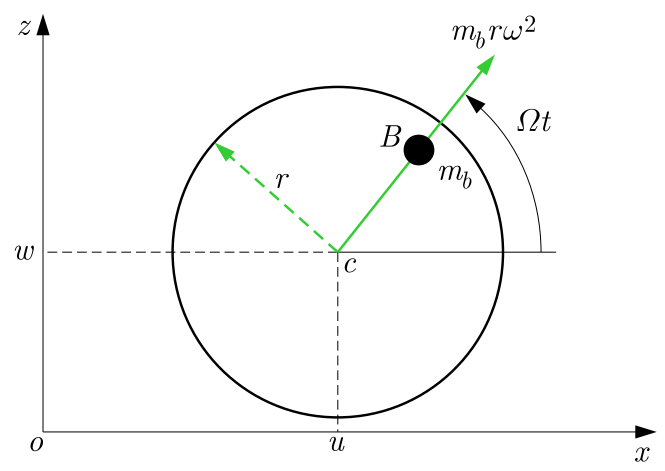

Fig. 2. Centrifugal forces due to unbalance

The coordinates of the unbalance in the fixed coordinate system are given by

$$
\begin{aligned}
& x(t)=u_{B}+d \cos \Omega t \quad \Rightarrow \quad \frac{x(t)}{d t}=u_{b}-d \Omega \sin \Omega t \\
& z(t)=w_{B}+d \sin \Omega t \Rightarrow \frac{z(t)}{d t}=w_{b}+d \Omega \cos \Omega t
\end{aligned}
$$

Its kinetic energy is given by

$$
T_{\text {Bal }}=\frac{1}{2} m_{b}\left[\left(\dot{u}_{B}^{2}+\dot{w}_{B}^{2}\right)+\Omega^{2} d^{2}+2 \Omega d \dot{u}_{B} \sin \Omega t-2 \Omega d \dot{w}_{B} \cos \Omega t\right]
$$

The term $\Omega^{2} d^{2} / 2$ is constant and will not intervene in the equations. The mass of the imbalance is negligible in comparison to the rotor mass; the expression of the kinetic energy can be approximated by

$$
T_{B a l} \approx m_{b} d \Omega\left(\dot{u}_{B} \sin \Omega t-\dot{w}_{B} \cos \Omega t\right)
$$

By introducing the generalized coordinates, it can be rewritten as follows

$$
T_{B a l} \approx m_{b} d \Omega\left(\dot{A}_{u} \dot{Y}_{B}^{3}+\dot{B}_{u} Y_{B}^{2}+\dot{C}_{u} Y_{B}^{2}+\dot{D}_{u}\right) \sin \Omega t-\left(\dot{A}_{w} \dot{Y}_{B}^{3}+\dot{B}_{w} Y_{B}^{2}+\dot{C}_{w} Y_{B}^{2}+\dot{D}_{w}\right) \cos \Omega t
$$

The unbalance generates strong vibrations at the operational rotation speed of the turbine. They appear when the gravity axis of the rotor (equilibrium mass axis) does not correspond to its axis 
of rotation. This unbalance is caused by an inhomogeneous distribution of mass around the axis of rotation. In the case of turbocharger stationary motion, where the bearing part is used to guide the rotating shaft and the radial load is small and comprised primarily of the unbalance, which may be reduced if necessary. On the other side, the ball bearing damage causes several major factors; such as surface material fatigue as a result of constraints that produce flaking and cracking. Furthermore, the superficial fatigue can be aggravated by some effects such as insufficient lubrication, surface conditions, shock. Also for the elements in contact, the wear can aggravate the unstable phenomena during the operation of the machine.

For a ball bearing, Fig. 3, it comprises $n_{b}$ number of balls, and rotates at the frequency $f_{r}$ (shaft rotation frequency). The presence of a vibration defect creates shocks between the rotating elements of the channel whose frequencies are defined by the expressions in equations (3.9) and (3.14).

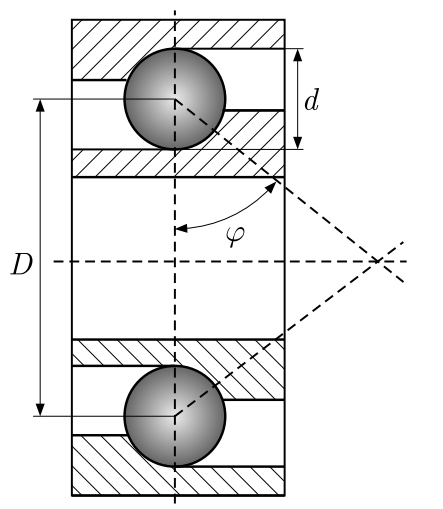

With D: ball center diameter, d: the ball diameter: $\varphi$ contact angle between the ball and the raceway

Fig. 3. Geometric characteristics of the bearing

The frequencies relative to the defects on each rotating elements are functions of the passage frequency of the rotating element in the outer ring. It is given by the following equation

$$
f_{b e}=\frac{f_{r} n_{b}}{2}\left(1-\frac{d}{D} \cos \phi\right)
$$

The passage frequency of the rotating element in the defected inner ring, is given by the following equation

$$
f_{b i}=\frac{f_{r} n_{b}}{2}\left(1+\frac{d}{D} \cos \phi\right)
$$

The defects frequency on the ball are given by

$$
f_{B}=\frac{f_{r} n_{b}}{2}\left[1-\left(\frac{d}{D} \cos \phi\right)^{2}\right]
$$

The passage frequency of the defected ball in the outer ring or in the inner ring is presented as follows

$$
f_{c}=\frac{f_{r}}{2}\left[1-\left(\frac{d}{D} \cos \phi\right)\right]
$$

Taking into account the modulation of the signal by the frequency $f_{c}$, the above equations are modified, so the defects in the inner ring become

$$
f_{b i}=\frac{f_{r} n_{b}}{2}\left(1+\frac{d}{D} \cos \phi\right) \pm f_{c}
$$


And the defects on the ball are expressed as

$$
f_{B}=\frac{f_{r} n_{b}}{2}\left[1-\left(\frac{d}{D} \cos \phi\right)^{2}\right] \pm f_{c}
$$

This signal modulation leads to functional modeling imperfections of the examined gas turbine. In the following Section, the proposed approach based on spectral analysis at a low range frequency will be applied to a practical case which is considered in a real machine in the presence of an unbalance anomaly.

\section{Industrial investigations}

In this study of the vibration behavior based on the analysis of measured signals on a gas turbine installed in the gas center of TIMZHERT, at Hassi R-Mel, south of Algeria, has been examined. Figure 4 shows the studied gas turbine with the specifications given in Table 1. This machine undergoes vibration effects observed during its operation. The measurements were carried out on this turbine via a mobile accelerometer for ensuring diagnosis of various vibration phenomena occuring at bearing No. 1 of the studied gas turbine. While guaranteeing the maintenance policy, vibrations are measured, processed and used for diagnosis to assess mechanical conditions of the examined turbine.

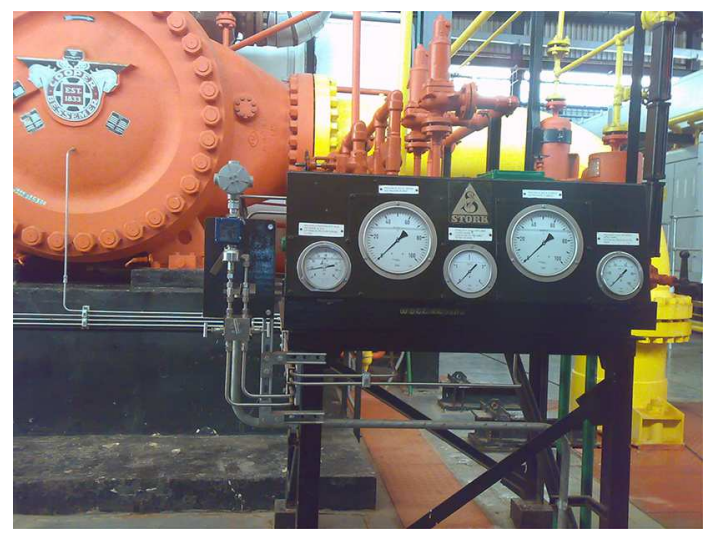

Fig. 4. Studied GE 3002 gas turbine

Table 1. Specifications of the examined turbine

\begin{tabular}{|l|c|}
\hline \multicolumn{1}{|c|}{ Parameter } & Value or symbol \\
\hline \hline Designer: General Electric & GE \\
\hline Type & MS 3002 \\
\hline Serial No. & 226293 \\
\hline HP speed axial compressor & $7100 \mathrm{tr} / \mathrm{min}$ \\
\hline No. floor wheel (s) HP & 01 \\
\hline No. axial compressor stage & 15 \\
\hline BP speed & $6500 \mathrm{tr} / \mathrm{min}$ \\
\hline No. BP wheel & 01 \\
\hline Turbine power $80 \%$ & $9400 \mathrm{CV}$ \\
\hline Fuel consumption rate $\left(100 \% \mathrm{H} 27^{\circ} \mathrm{C}\right)$ & $3.84 \mathrm{~m}^{3} / \mathrm{h}$ \\
\hline Pressure of exhaust gas & $1009.3 \mathrm{bars}$ \\
\hline global peas & $63000 \mathrm{k}$ \\
\hline
\end{tabular}




\subsection{Applications results}

In this Section, the obtained experimental results using the measurements performed on the studied gas turbine are presented. The results were performed on each bearing (bearing 1 and 4) in the vertical direction and with a rotation speed of the shaft $\omega=7100 \mathrm{tr} / \mathrm{min}$. The characteristic frequencies of the bearing defects are listed in Table 2. The values given in Table were drawn on the basis of dimensions of the bearing and on the basis of rotation frequency of the shaft, using the frequency $f_{r}=V(\operatorname{tr} / \mathrm{min}) / 60 \mathrm{~s}=7100 / 60=118.33 \mathrm{~Hz}$ (where $V$ is the shaft rotation speed and $n_{b}=15$ is the number of beads).

Table 2. Characteristic frequencies of bearing defects

\begin{tabular}{|c|c|c|}
\hline$f_{r}[\mathrm{~Hz}]$ & $f_{b e}[\mathrm{~Hz}]$ & $f_{b i}[\mathrm{~Hz}]$ \\
\hline \hline 118.33 & 748 & 1029 \\
\hline
\end{tabular}

According to the obtained results of measurements, it is found that the measured values on the turbine at bearing 1 indicate the presence of vibrations in the vertical direction over a range of frequencies $(0-10000 \mathrm{~Hz})$. In order to diagnose this vibration failure, a spectral analysis has been carried out. The obtained experimental results using the experimental conditions show the amplitude of the signal versus the frequency, see Figs. 5 and 5b. It is important to clarify that the bearing life depends on the load acting on the shaft, on the rotational speed and on the point of application of the force.

(a)
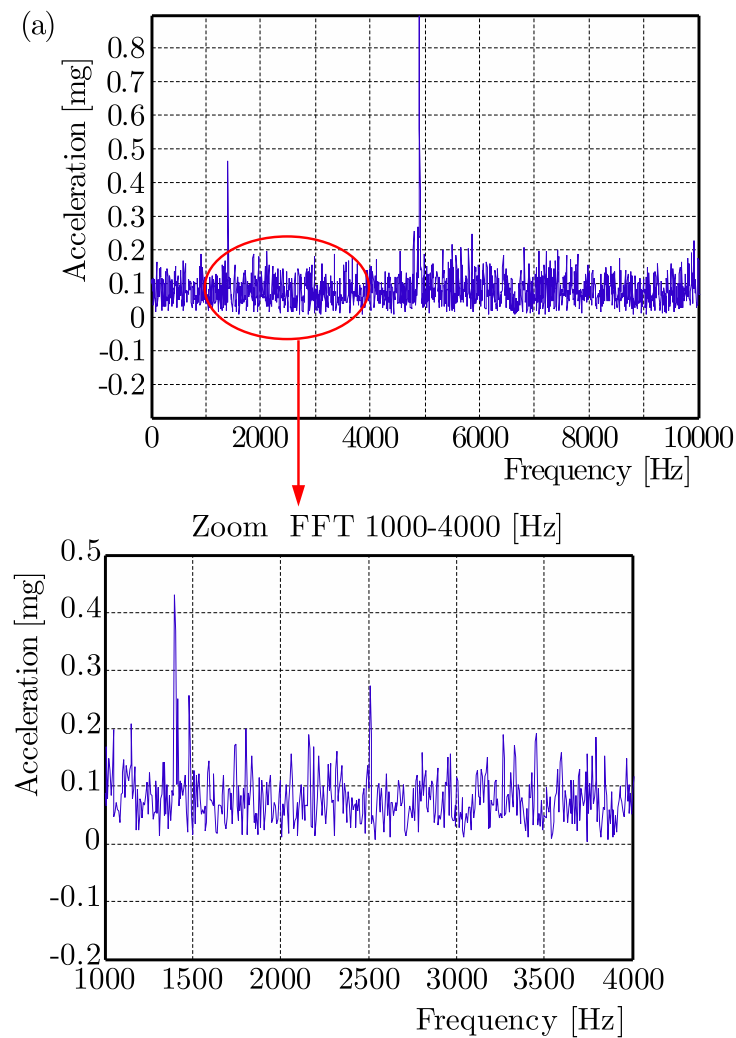

(b)
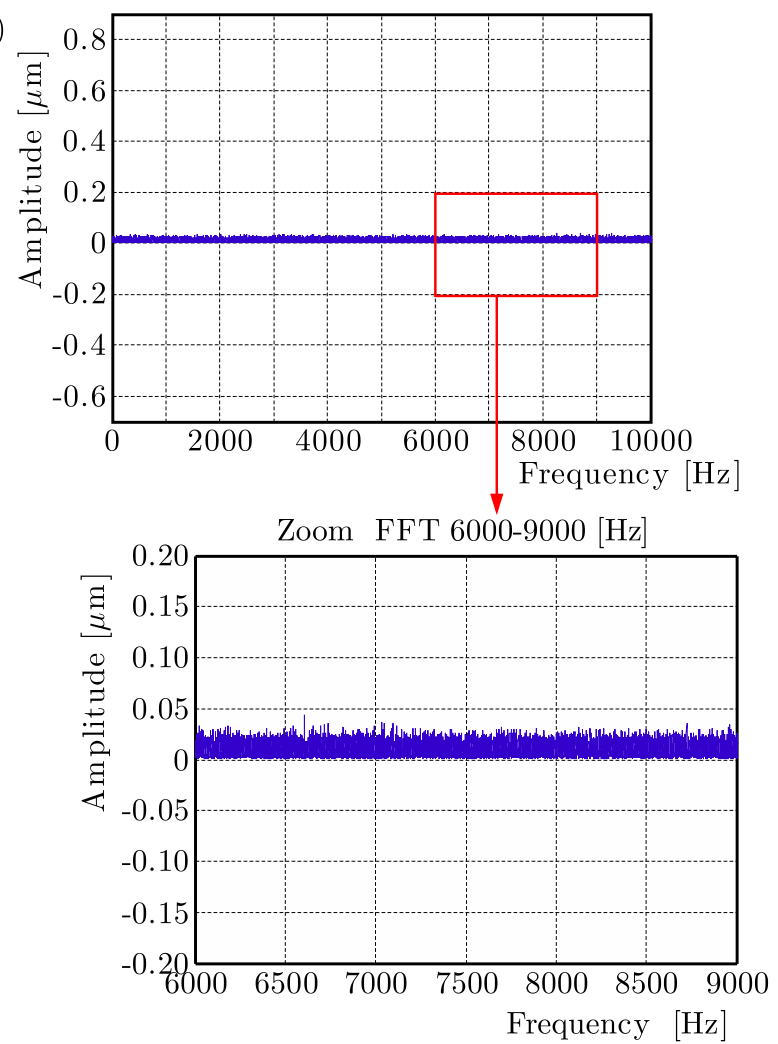

Fig. 5. Vibration signal: (a) of a defected bearing, (b) of a bearing with a fault located in the inner ring

The gas turbine is operated by a set of commands that support the machine during starting and accelerating till the loading phase. This automatic control helps to maintain the system to be stable. The measurements of variables such as pressure, velocity and acceleration are performed using an instrumentation device, as shown in Fig. 6. 


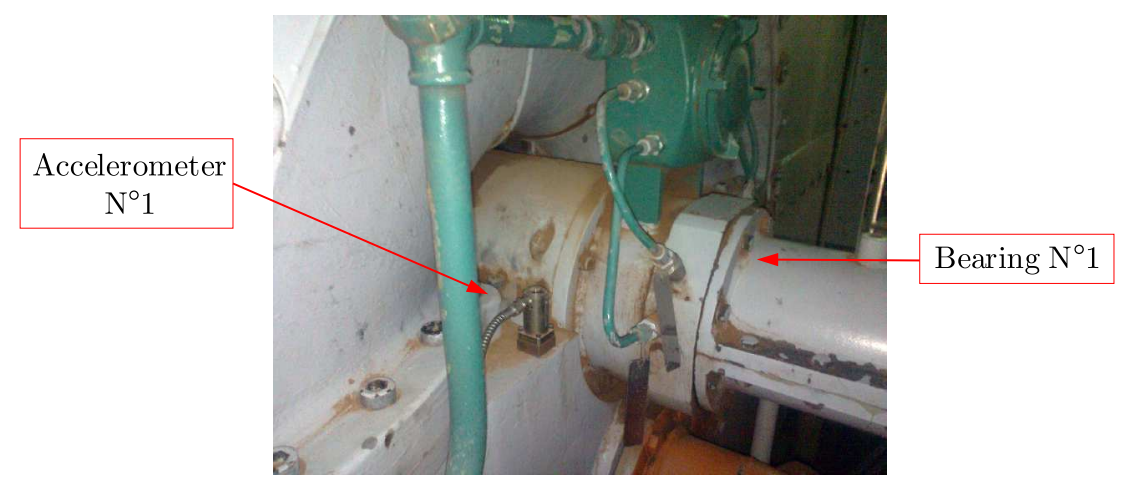

Fig. 6. Position of installed sensors in the studied gas turbine

Analysis of various elements of the studied gas turbine was carried out on this installation, starting from the study of rotation speed and its frequency, which must define the parameters useful for the proposed monitoring system. This is based on the knowledge of variations of the expected unstable phenomena, given by characteristic frequencies of possible defects such as unbalance, the alignment and the attachment. Also, the resulting vibrations due to forces developed or transmitted by the bearings caused by the unbalance have been analyzed. To analyze these phenomena, Movilog 2 with FFT mono channel and the accelerometer with speed of $100 \mathrm{mV} / \mathrm{g}$ type (HS-AM004) are used, see Figs. 7a and 7b.

(a)

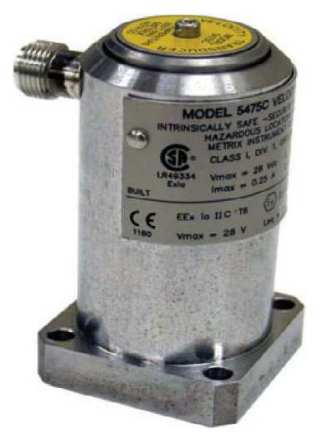

(b)

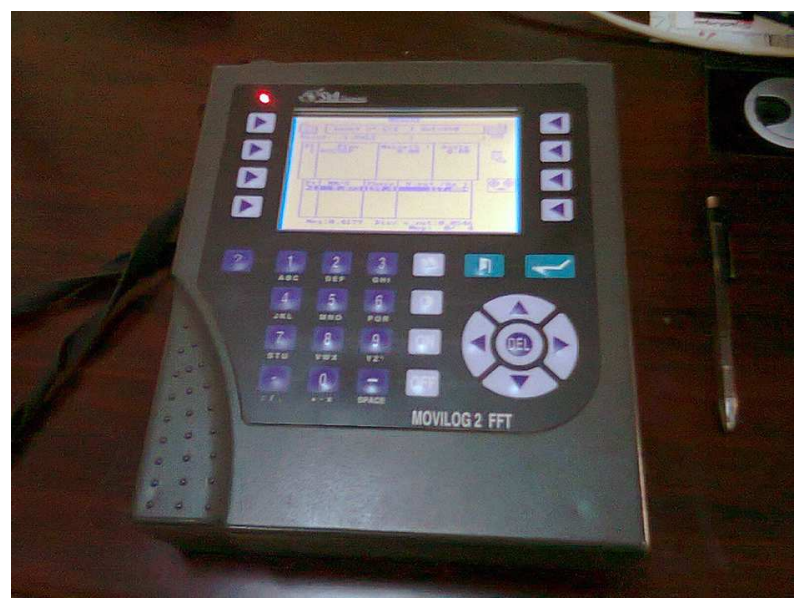

Fig. 7. (a) Used accelerometer; (b) Movilog 2 with FFT mono channel

This is done with the input of balancing data for the selected rotation speed ( $V=118 \mathrm{rpm})$, rotor mass $(M=3400 \mathrm{~kg})$ and rotor radius $(R=75 \mathrm{~mm})$. At the rotational speed of $7100 \mathrm{rpm}$, where $f_{r}=118.33 \mathrm{~Hz}$ in the frequency range of $0-10000 \mathrm{~Hz}$, the large amplitude peaks are observed, where $f_{r}$ is the rotation frequency of the shaft as shown in Fig. 8. This figure is the continuous display of the spectral density of the measured vibration signal within frequency regions corresponding to proper modes of the inner ring.

The appearance of such components as the shaft of the turbine (HP rotor) runs with the defected bearing is explained by the presence of oscillations in the torque load. These fluctuations have the feature to occur at the same characteristic frequency of the defect. In the present diagnostic method, the appearance of these components is used as a reference for diagnosing the presence of the anomalies in the studied gas turbine.

The levels shown in Tables 3 present the overall levels from the accelerometers within the frequency ranges $(1000 \mathrm{~Hz}-10000 \mathrm{~Hz})$. 


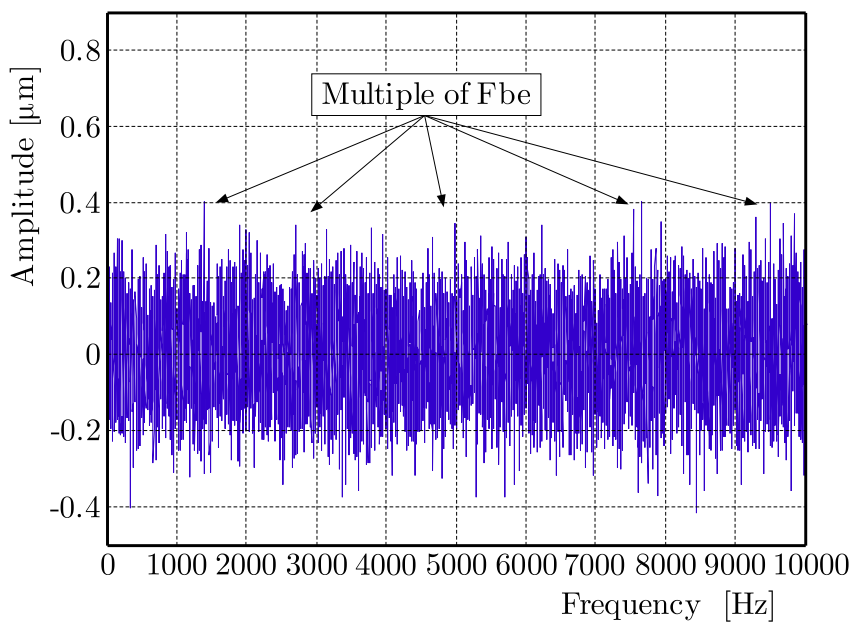

Fig. 8. Vibration signal of the bearing with a fault located in the outer ring

Table 3. Overall levels from accelerometers of the studied gas turbine

\begin{tabular}{|c|c|c|c|c|c|}
\hline \multicolumn{2}{|c|}{ SC1 } & Kurtosis & $\begin{array}{c}\text { Crete } \\
\text { factor }\end{array}$ & $\begin{array}{c}\text { Acc. }[\mathrm{mg}] \\
10000 \mathrm{~Hz}\end{array}$ & $\begin{array}{c}\text { Speed [mm/s] } \\
1000 \mathrm{~Hz} \text { Pic }\end{array}$ \\
\hline \hline \multirow{3}{*}{} & \multirow{3}{*}{$\mathrm{RV}$} & 3.0 & 4.6 & 208.8 & 2.9 \\
\cline { 3 - 6 } & 6.9 & 7.0 & 89.8 & 1.3 \\
\hline & & 2.9 & 4.5 & 61.8 & 2.6 \\
\hline \multirow{3}{*}{$\mathrm{HP}$} & \multirow{3}{*}{$\mathrm{RH}$} & 3.0 & 5.0 & 32.0 & 0.2 \\
\cline { 3 - 6 } & & 7.0 & 7.0 & 13.3 & 0.1 \\
\hline & & 2.9 & 5.2 & 7.0 & 0.2 \\
\hline \multirow{3}{*}{$\mathrm{AX}$} & 2.7 & 4.5 & 160.6 & 0.7 \\
\cline { 3 - 6 } & 6.3 & 7.1 & 69.9 & 0.2 \\
\cline { 3 - 6 } & 2.6 & 4.4 & 45.6 & 0.7 \\
\hline
\end{tabular}

(a)

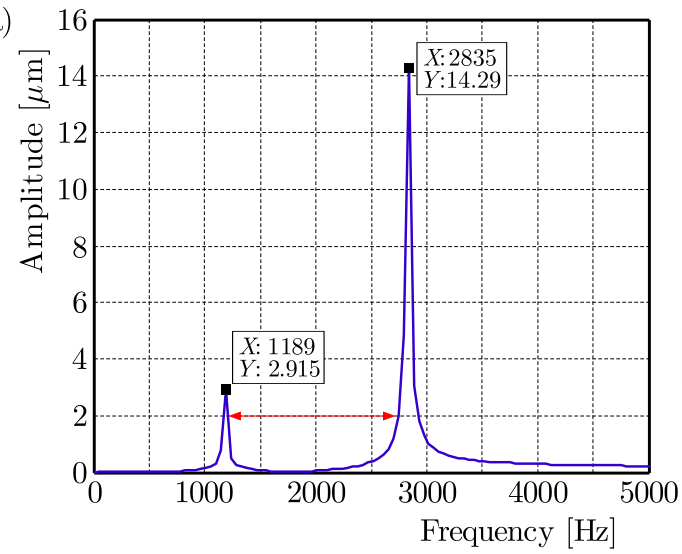

(b)

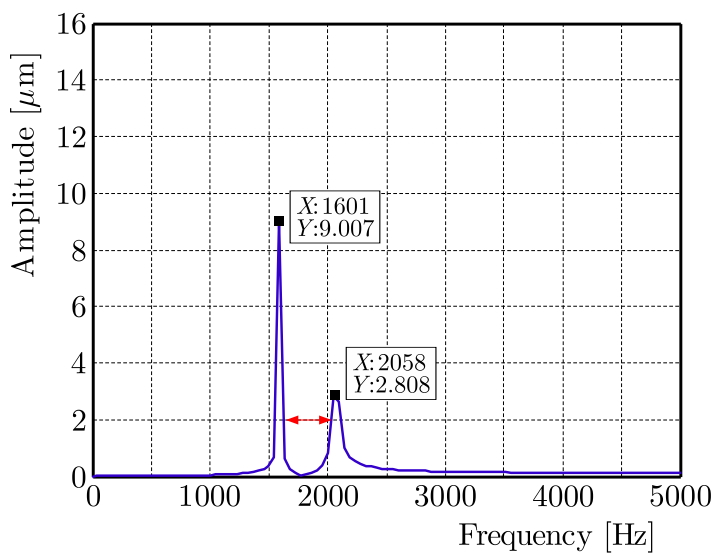

Fig. 9. Amplitude of the unbalance response: (a) test 1, (b) test 2

For unbalance defects, the operator can compensate for this misalignment by adding or removing known masses at specific locations on the rotor. These imbalance defects are mainly due to the manufacturing errors. They can explained by the presence of poor homogeneity of the materials used in the rotor structure. In order to verify the capacity of the system to balancing strong unbalances, additional balancing tests were carried out. Figures 9a,b and 10a,b show results of tests with two balancing speeds, the main goal is to reduce the overall vibration levels at all speeds and to kept the amplitude of vibration to be stable. 
(a)

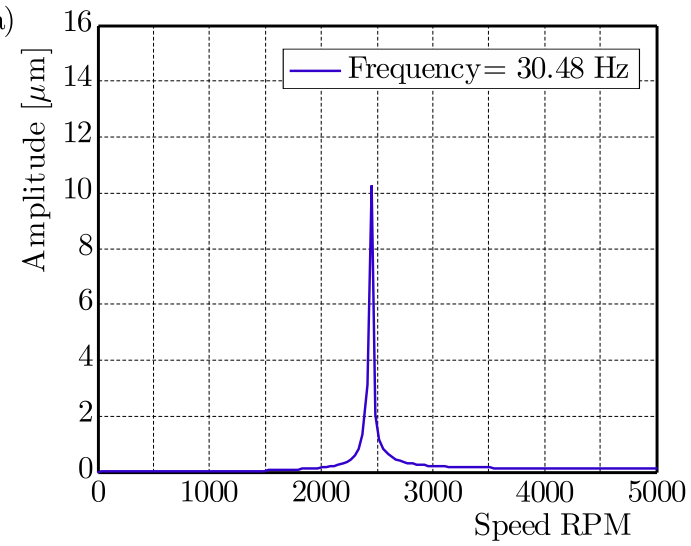

(b)

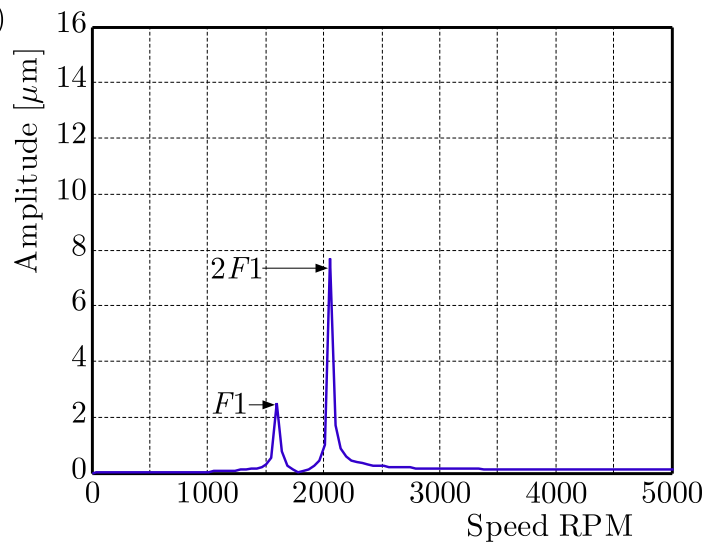

Fig. 10. Amplitude of the unbalance response: (a) test 3, (b) test 4

A misalignment is occurred when the peak amplitude is captured, generally up to 2 or 3 times the rotation frequency. The vibration measurements are in function of choice of the measurement points. In the studied case, the bearings are chosen as the points of measurement and data collecting on the examined gas turbine. Figures 11a and 11b successively present the evolution of the spectral structure (time signal) when a fault alignment is occurred.

(a)

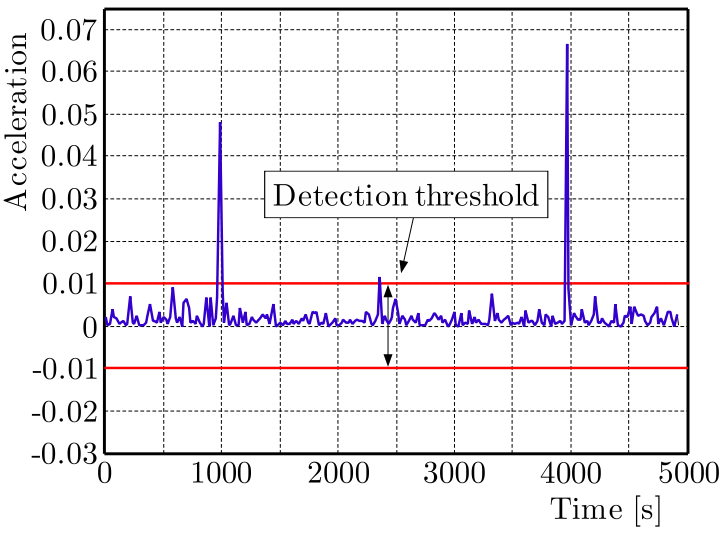

(b)

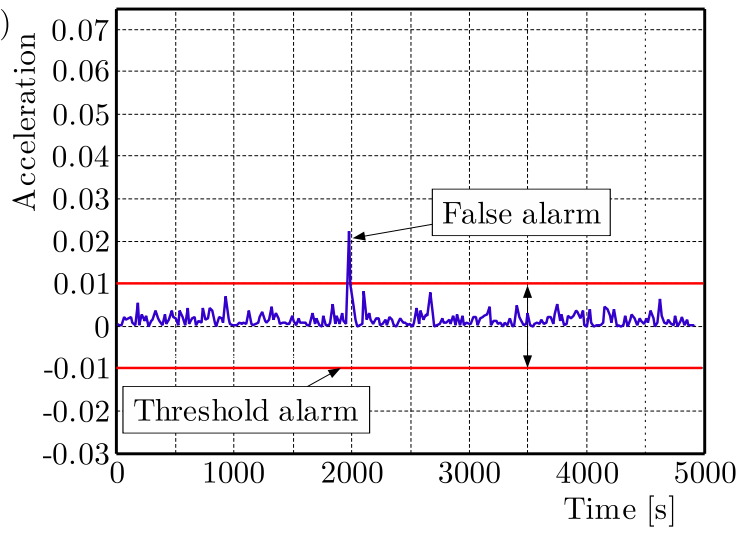

Fig. 11. (a) Time measurement signal of the acceleration in the presence of alignment defects;

(b) temporal signal without defects

At a speed of $7100 \mathrm{rpm}$, for a frequency range of $0-5000 \mathrm{~Hz}$, a peak is observed (the shaft rotation frequency) which characterizes the misalignment fault. In Fig. 11b, the fault is directional, which is the case of the detected and identified misalignment on the gas turbine rotor. The results obtained were used to analyze the signature of an axial vibration fault. Unlike unbalanced, this defect is often seen in the axial direction.

\section{Conclusion}

The understanding of the dynamic vibration behavior in gas turbines allows identification and localization of the source of the vibration anomaly as well as quantification of the defect. In this paper, the rotor dynamics is studied to ensure the improvement of security and performance of the studied system. The developed model allows one to analyze the dynamic behavior of a HP turbine rotor type MS 3002 rotating at a high speed and supported by bearings having defects that are presenting instabilities areas varying with the rotation frequency and creating furthermore new critical frequencies. On the other side, it is shown that some important effects 
can occur. Firstly, the wear of components regarding the rotor elements or mass distribution which can generate new critical speeds. Secondly, the defect in the inner ring of a bearing can generate the passing frequency which varies with the speed of rotation. Thirdly, the alignment effect manifests itself as a large amplitude component of the rotation frequency of the rotor in the radial direction, sometimes in the axial direction in the case of a rotor cantilever.

\section{References}

1. Ali U., Font Palma C., Hughes K.J., Ingham D.B., Ma L., Pourkashanian M., 2015, Impact of the operating conditions and position of exhaust gas recirculation on the performance of a micro gas turbine, Computer Aided Chemical Engineering, 37, 2417-2422

2. Ashour O., Khalidi A., Fadlun E., Giannini N., Pieri M., Ceccherini A., 2012, On-line monitoring of gas turbines to improve their availability, reliability, and performance using both process and vibration data, Proceeding of the 3rd Gas Processing Symposium, 334-343

3. Djaidir B., Hafaifa A., Kouzou A., 2015, Monitoring gas turbines using speedtronic Mark VI Control Systems, Pipeline and Gas Journal, 242, 10, 48-86

4. Djaidir B., Hafaifa A., Kouzou A., 2016, Vibration detection in gas turbine rotor using artificial neural network, International Conference on Acoustics and Vibration ATAVI'16, March 21-23, 2016, Hammamet-Tunisia

5. Djeddi A.Z., Hafaifa A., Kouzou A., Abudura S., 2016, Exploration of reliability algorithms using modified Weibull distribution: Application on gas turbine, International Journal of System Assurance Engineering and Management, DOI 10.1007/s13198-016-0480-9, First online: 12 May 2016, 1-10

6. Djeddi A.Z., Hafaifa A., Salam A., 2015, Gas turbine reliability model based on tangent hyperbolic reliability function, Journal of Theoretical and Applied Mechanics, 53, 3, 723-730

7. Eshati S., Abu A., Laskaridis P., Khan F., 2013, Influence of water-air ratio on the heat transfer and creep life of a high pressure gas turbine blade, Applied Thermal Engineering, 60, 1-2, $335-347$

8. Ford C.L., Carrotte J.F., Walker A.D., 2013, The application of porous media to simulate the upstream effects of gas turbine injector swirl vanes, Computers and Fluids, 77, 143-151

9. Galindo J., Fajardo P., Navarro R., Garca-Cuevas L.M., 2013, Characterization of a radial turbocharger turbine in pulsating flow by means of CFD and its application to engine modeling, Applied Energy, 103, 116-127

10. Guasch A., Quevedo J., Milne R., 2000, Fault diagnosis for gas turbines based on the control system, Engineering Applications of Artificial Intelligence, 13, 4, 477-484

11. Guemana M., Hafaifa A., Rahmoune M.B., 2015, Reliability study of gas turbines for improving their availability by ensuring optimal exploitation, OIL GAS European Magazine, 2, 88-91

12. GüNYAZ A., 2013, A modeling and control approach to advanced nuclear power plants with gas turbines, Energy Conversion and Management, 76, 899-909

13. Hadroug N., Hafaifa A., Kouzou A., Chaibet A, 2016, Faults detection in gas turbine using hybrid adaptive network based fuzzy inference systems to controlling there dynamic behavior, Diagnostyka - The Journal of Polish Society of Technical Diagnostics (PSTD), 17, 4, 3-17

14. Jagaduri R.T., Radman G., 2007, Modeling and control of distributed generation systems including PEM fuel cell and gas turbine, Electric Power Systems Research, 77, 1, 83-92

15. Jurado F., Carpio J., 2006, Improving distribution system stability by predictive control of gas turbines, Energy Conversion and Management, 47, 18/19, 2961-2973

16. Kim K.H., Ko H.-J., Perez-Blanco H., 2011, Analytical modeling of wet compression of gas turbine systems, Applied Thermal Engineering, 31, 5, 834-840 
17. Krzyzynski T., Popp K., Sextro W., 2000, On some irregularities in dynamic response of cyclic periodic structures, Chaos, Solitons and Fractals, 11, 1597-1609

18. Lee M.C., Chung J.H., Park W.S., Park S., Yonn Y., 2013, The combustion tuning methodology of an industrial gas turbine using a sensitivity analysis, Applied Thermal Engineering, 50, 1, 714-721

19. Liu X., Lu C., Liang S., Godbole A., Chen Y., 2015, Influence of the vibration of large-scale wind turbine blade on the aerodynamic load, Energy Procedia, 75, 873-879

20. Madhavan S., Rajeev J., Sujatha C., Sekha A.S., 2014, Vibration based damage detection of rotor blades in a gas turbine engine, Engineering Failure Analysis, 46, 26-39

21. Nikpey H., Assadi M., Breuhaus P., Mørkved P.T., 2014, Experimental evaluation and ANN modeling of a recuperative micro gas turbine burning mixtures of natural gas and biogas, Applied Energy, 117, 30-41

22. Rahmoune M.B., Hafaifa A., Guemana M., 2015, Neural network monitoring system used for the frequency vibration prediction in gas turbine, The 3rd International Conference on Control, Engineering and Information Technology CEIT'2015, 25-27 May 2015 Tlemcen, Algeria

23. SAnaye S., TAhani M., 2010, Analysis of gas turbine operating parameters with inlet fogging and wet compression processes, Applied Thermal Engineering, 30, 2/3, 234-244

24. Sextro W., Popp K., Krzyzynski T., 2001, Localization in nonlinear mistuned systems with cyclic symmetry, Nonlinear Dynamics, 25, 207-220

25. Simani S., Patton R.J., 2008, Fault diagnosis of an industrial gas turbine prototype using a system identification approach, Control Engineering Practice, 16, 7, 769-786 